\title{
SIDE EFFECTS OF INTRAVENOUS GANCICLOVIR AND COMBINATION OF INTRAVENOUS GANCICLOVIR-ORAL VALGANCICLOVIR IN PEDIATRIC PATIENTS WITH CYTOMEGALOVIRUS INFECTION
}

\author{
I WAYAN ANDREW HANDISURYA*, NELLY AMALIA RISAN, DWI PRASETYO
}

Department of Child Health, Hasan Sadikin General Hospital, Bandung, Indonesia. Email: andrew.ika2015@gmail.com

Received: 01 June 2019, Revised and Accepted: 07 July 2019

\section{ABSTRACT}

Objective: Intravenous ganciclovir and oral valganciclovir are known to have severe side effects against hematopoiesis and kidney function. This study aims to look for these side effects in pediatric patients with cytomegalovirus (CMV) infection treated with ganciclovir or a combination regimen of both in Hasan Sadikin General Hospital, a tertiary care center in West Java, Indonesia.

Methods: This was a cross-sectional study using medical records and laboratory examination data from pediatric patients with CMV infection who received ganciclovir of $6 \mathrm{mg} / \mathrm{kg}$ twice a day, valganciclovir of $16 \mathrm{mg} / \mathrm{kg}$ twice a day, or a combination of both in Hasan Sadikin General Hospital from January 2015 to January 2019. Differences before and after therapy were analyzed to look for any side effects in each regimen and duration of therapy.

Results: Out of 115 patients, there were 53 subjects who met the research criteria. Majority of the subjects used a combination regimen ( $\mathrm{n}=29,55 \%$ ) for 6 weeks $(n=36,68 \%)$. There was no statistically significant difference $(p>0.05)$ in the incidence of anemia, neutropenia, thrombocytopenia, increase in creatinine serum, or increase in blood urea nitrogen for different regimen or duration of therapy.

Conclusion: No side effects were found to be related with therapeutic regimen or duration of therapy in pediatric patients with CMV infection in this study.

Keywords: Ganciclovir, Valganciclovir, Combination regimen, Antiviral, Cytomegalovirus infection, Side effects, Pediatrics.

(c) 2019 The Authors. Published by Innovare Academic Sciences Pvt Ltd. This is an open access article under the CC BY license (http://creativecommons. org/licenses/by/4. 0/) DOI: http://dx.doi.org/10.22159/ajpcr.2019.v12i9.34410

\section{INTRODUCTION}

Cytomegalovirus(CMV) is a double-stranded deoxyribonucleic acid (DNA) virus that belongs to the Herpesviridae family and may infect any age group [1]. The prevalence of CMV infection is said to be higher in children with low socioeconomic status and those living in developing countries [2,3]. Delay in management may cause bad outcomes and poor prognosis in these patients $[4,5]$.

Ganciclovir is an antiviral that works by preventing CMV DNA replication. Intravenous ganciclovir therapy for 6 weeks provides shortterm clinical improvement and better neurological development [6-8]. Valganciclovir is the valine ester form of ganciclovir which will be converted into its active form in intestinal and liver epithelial cells $[9,10]$. Oral valganciclovir is said to have the same efficacy and pharmacokinetic properties with intravenous ganciclovir [7,11-13].

Intravenous ganciclovir and oral valganciclovir combination therapy regimen can be considered if there is difficulty in administering intravenous ganciclovir for 6 weeks [7]. The use of long-term peripheral venous catheter and higher treatment cost is several considerations for Hasan Sadikin General Hospital, a tertiary care center in Bandung, West Java, Indonesia, to begin implementing combination regimen for pediatric patients with CMV infection $[11,12]$.

Intravenous ganciclovir, oral valganciclovir, or a combination of both with the total duration of 6 weeks are recommended as the therapy for CMV infection with central nervous system involvement or severe focal organ disease $[7,14]$. Patients with CMV infection in developing countries, such as Indonesia, usually present late and are associated with many comorbidities; therefore, giving therapy in these patients requires a lot of consideration. Both of these antiviral agents are known to have high toxicity; unfortunately, studies for their side effects are still a few, with limited population and varying results $[11,12,15,16]$. The most common side effects are myelosuppression of hematopoiesis, such as neutropenia, thrombocytopenia, and anemia [7,17-19]. Pharmacologically, ganciclovir has a toxic effect on the kidney, so it is necessary to monitor kidney function $[7,17,18]$.

As far as we know, there have been no studies of the side effects of ganciclovir, valganciclovir, or combination regimen done in developing countries; thus, this study aims to look for these side effects in pediatric patients with CMV infection admitted to Hasan Sadikin General Hospital to become the basis for clinical guidance of pediatric CMV infection in developing countries.

\section{METHODS}

The design of this study was cross-sectional. The study was conducted in May 2019 by searching the Hospital Information System database to find data on patients aged 0-18 years with CMV infection who received ganciclovir and/or valganciclovir from January 2015 to January 2019. Out of 115 patients found in the database, only 91 patients and their respective medical records were found.

The inclusion criteria for this study were as follows: Patients diagnosed with CMV infection using reactive CMV immunoglobulin $\mathrm{M}$; patients treated with intravenous ganciclovir of $6 \mathrm{mg} / \mathrm{kg} 2$ times a day, or oral valganciclovir of $16 \mathrm{mg} / \mathrm{kg}$ twice a day, or a combination of both; and patients having more than 1 laboratory examination during the course of the treatment. The exclusion criteria were as follows: Patients who had shown clinical manifestations of cirrhosis before the therapy, patients with immunodeficiency condition (such as primary immunodeficiency disease, HIV/AIDS, malignancy, receiving chemotherapy, or having organ transplant history), and patients who died during the treatment course with CMV infection excluded from the cause of death. 
The side effects of therapy related to myelosuppression of hematopoiesis were anemia (hemoglobin $<11 \mathrm{~g} / \mathrm{dL}$ ), neutropenia (neutrophil $<1500 / \mathrm{mm}^{3}$ ), and thrombocytopenia (thrombocyte $<150,000 / \mathrm{mm}^{3}$ ). The side effects associated with decreased kidney function were sought in the form of an increase in creatinine serum above $0.5 \mathrm{mg} / \mathrm{dL}$ and an increase in blood urea nitrogen above $20 \mathrm{mg} / \mathrm{dL}$. The data were then inputted in the "Statistical Product and Service Solution" program for Windows version 22.0. Univariable analysis was conducted to describe the characteristics of the subject. The data were presented in numbers and percentages for categorical data and in mean, standard deviation, median, minimum, and maximum values for numerical data. The normality of the data was tested using Kolmogorov-Smirnov test before doing the multivariable analysis. To analyze differences before and after therapy, one-way analysis of variance (ANOVA) test was used for normally distributed data and Kruskal-Wallis test for abnormally distributed data. If one-way ANOVA or Kruskal-Wallis test obtained significant results, a post hoc analysis was performed. To analyze differences in compliance and side effects between regimens, Chisquare and Fisher's exact tests were used. The significance of the test results was determined based on the value of $\mathrm{p} \leq 0.05$.

Ethical clearance was obtained from the Research Ethics Committee of Hasan Sadikin General Hospital. The committee provided ethical clearance approval to conduct this study in the hospital. All data analyzed were further anonymized.

\section{RESULTS}

As shown in Fig. 1, of 91 medical records of patients treated with ganciclovir and/or valganciclovir from January 2015 to January 2019, there were 38 patients who did not meet the study criteria; therefore, the total subjects included in the study were 53 patients.

As shown in Table 1, majority of the subjects were male $(n=32,60 \%)$, aged $1-3$ months $(n=34,64 \%)$, had moderate malnutrition $(n=20,38 \%)$, microcephaly $(n=38,72 \%)$, and also involvement of the hepatobiliary system in the form of cholestasis $(n=39,74 \%)$. Out of 20 patients screened with head ultrasonography, seven patients had intracranial calcification and four patients had hydrocephalus. Out of 22 patients screened for hearing, 15 patients were planned for further examination and seven patients had already been examined with Brainstem Evoked Response Audiometry (BERA). Six of those examined with BERA were diagnosed with sensorineural hearing loss. Out of 17 patients who underwent eye screening, five patients were planned for further examination and six patients had abnormal results (three patients with cortical visual impairment, one patient with retinal scarring, one patient with immature retina, and one patient with vasculitis).

Most of the subjects used combination regimen $(n=29,55 \%)$ for 6 weeks $(n=36,68 \%)$. Two patients used valganciclovir regimen for
6 weeks due to unavailability of intravenous ganciclovir medication at that time. Since the sample size was too small, valganciclovir regimen group was excluded from further statistical analysis. Out of 17 patients experiencing treatment dropout before 6 weeks, two patients received therapy for 2 weeks, nine patients received therapy for 3 weeks, and four patients received therapy for 4 weeks.

The incidence of anemia increased from 58\% (n=30) at baseline to $70 \%(n=31)$ at week 2 . The incidence of neutropenia increased from $6 \%(\mathrm{n}=3)$ at baseline to $14 \%(\mathrm{n}=6)$ in week 1 . The incidence of thrombocytopenia increased from $8 \%(n=4)$ at baseline to $10 \%(n=5)$ in week 1 and increased furthermore to $14 \%(n=4)$ in week 6 . There was no statistically significant difference $(p>0.05)$ in the incidence of myelosuppression-related side effects of therapy between each regimen during the monitoring period of the therapy as shown in Table 2 .

The incidence of an increase in creatinine serum was $3 \%(n=1)$ at baseline and became $10 \%(n=2)$ in week 6 . The incidence of an increase in blood urea nitrogen was $12 \%(n=5)$ at baseline and became $45 \%$

Table 1: Study subject's characteristics

\begin{tabular}{ll}
\hline Characteristics & $\mathbf{n = 5 3 ( 1 0 0 \% )}$ \\
\hline Age group (months) & $1(2)$ \\
$<1$ & $34(64)$ \\
$1-3$ & $16(30)$ \\
$4-12$ & $2(4)$ \\
$>12$ & \\
Sex & $32(60)$ \\
Male & $21(40)$ \\
Female & \\
Nutritional status & $18(34)$ \\
Normal & $20(38)$ \\
Moderate malnutrition & $15(28)$ \\
Severe malnutrition & $14(26)$ \\
Head circumference & $38(72)$ \\
Normocephaly & $1(2)$ \\
Microcephaly & \\
Macrocephaly & $39(74)$ \\
Hepatobiliary involvement & $24(45)$ \\
Cholestasis & $10(19)$ \\
Hepatomegaly & \\
Splenomegaly & $22(41)$ \\
Therapy regimen & $2(4)$ \\
Ganciclovir & $29(55)$ \\
Valganciclovir & \\
Combination & $36(68)$ \\
Therapy compliance & $17(32)$ \\
Completion of therapy in 6 weeks & \\
Dropout from therapy before 6 weeks & \\
\hline &
\end{tabular}

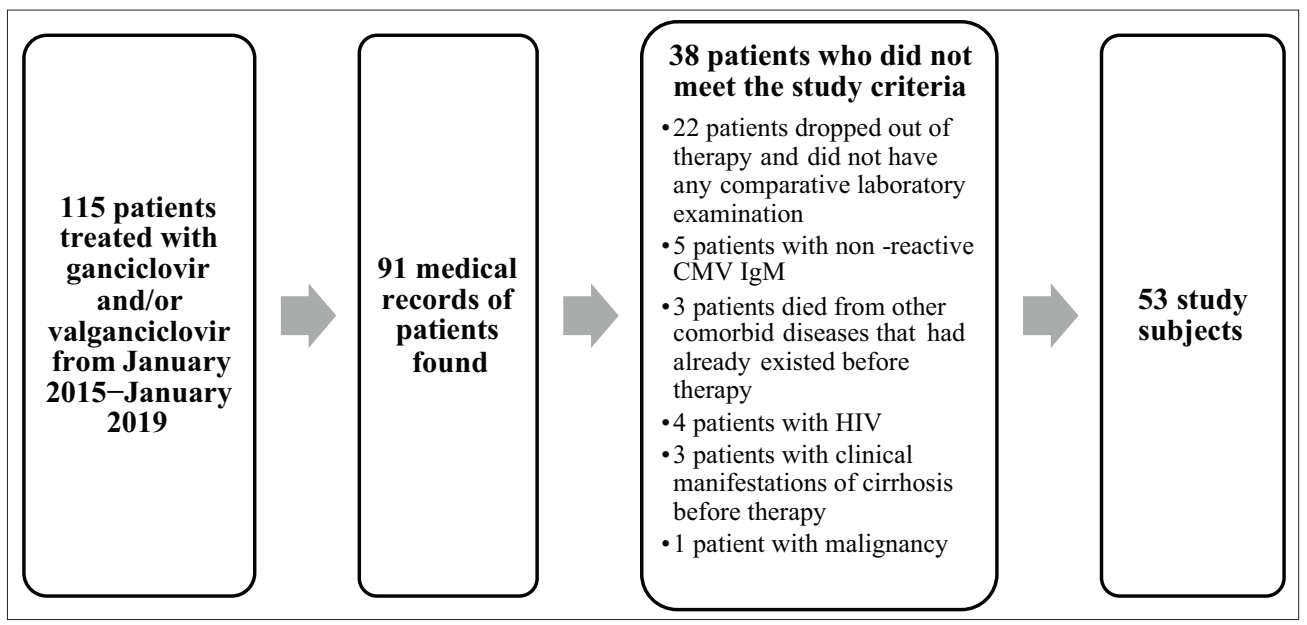

Fig. 1: Selection of study subjects 
Table 2: Correlation of myelosuppression-related side effects of therapy with the duration and therapeutic regimen

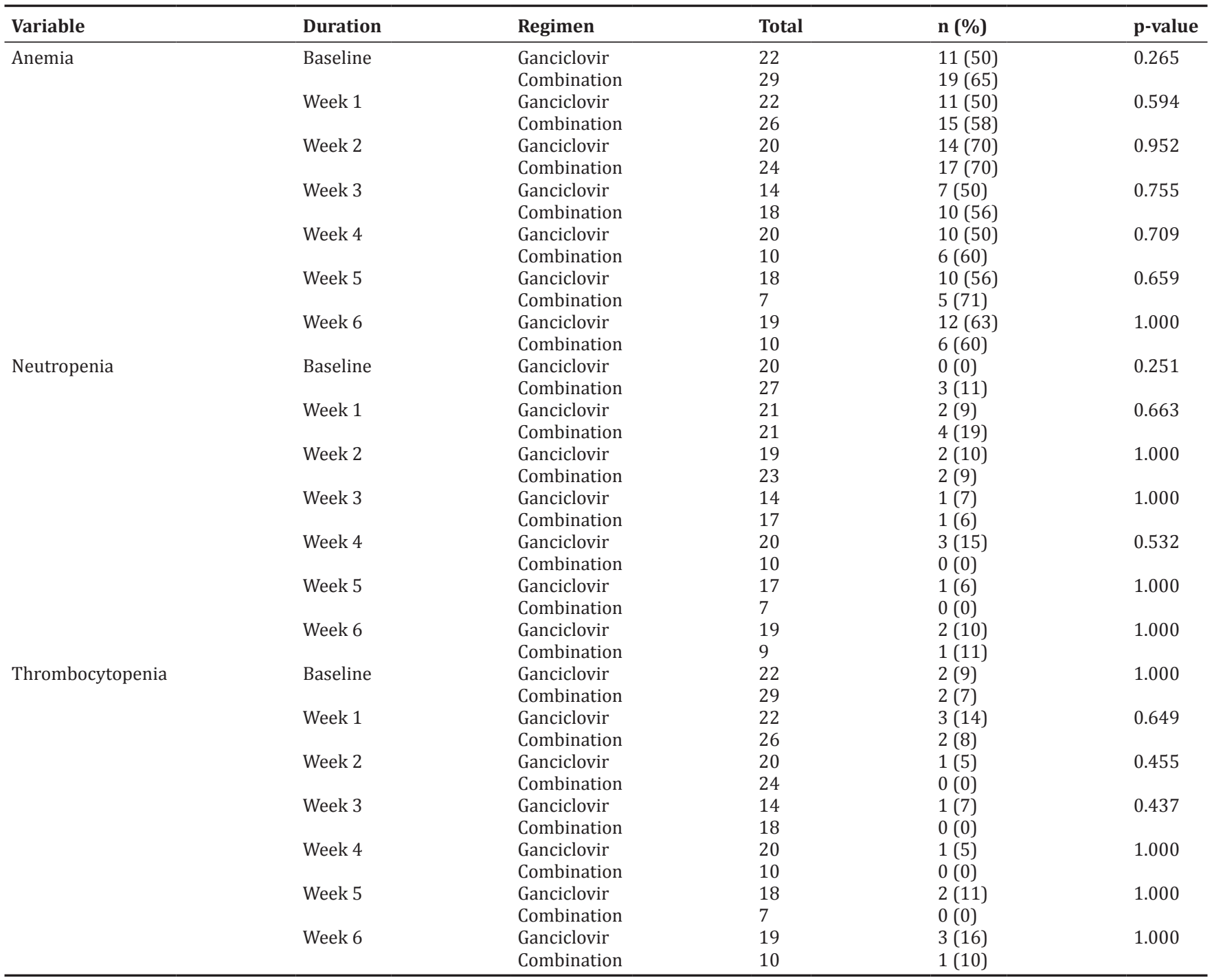

Description: Analysis was done using Chi-square test and Fisher's exact test

Table 3: Correlation of side effects of therapy related to the decrease in kidney function with the duration and therapeutic regimen

\begin{tabular}{|c|c|c|c|c|c|}
\hline Variable & Duration & Regimen & Total & n (\%) & p-value \\
\hline \multirow{4}{*}{$\begin{array}{l}\text { Increase in } \\
\text { creatinine serum }\end{array}$} & \multirow[t]{2}{*}{ Baseline } & Ganciclovir & 15 & $1(7)$ & \multirow[t]{2}{*}{0.385} \\
\hline & & Combination & 24 & $0(0)$ & \\
\hline & \multirow[t]{2}{*}{ Week 6} & Ganciclovir & 13 & $1(8)$ & \multirow[t]{2}{*}{1.000} \\
\hline & & Combination & 6 & 1 (17) & \\
\hline \multirow{14}{*}{$\begin{array}{l}\text { Increase in blood } \\
\text { urea nitrogen }\end{array}$} & \multirow[t]{2}{*}{ Baseline } & Ganciclovir & 16 & $2(12)$ & \multirow[t]{2}{*}{1.000} \\
\hline & & Combination & 24 & $3(12)$ & \\
\hline & \multirow[t]{2}{*}{ Week 1} & Ganciclovir & 13 & $3(23)$ & \multirow[t]{2}{*}{0.648} \\
\hline & & Combination & 14 & $2(14)$ & \\
\hline & \multirow[t]{2}{*}{ Week 2} & Ganciclovir & 18 & $4(22)$ & \multirow[t]{2}{*}{0.711} \\
\hline & & Combination & 17 & 5 (29) & \\
\hline & \multirow[t]{2}{*}{ Week 3} & Ganciclovir & 6 & $2(33)$ & \multirow[t]{2}{*}{1.000} \\
\hline & & Combination & 12 & $3(25)$ & \\
\hline & \multirow[t]{2}{*}{ Week 4} & Ganciclovir & 16 & $6(50)$ & \multirow[t]{2}{*}{0.670} \\
\hline & & Combination & 24 & 3 (37) & \\
\hline & \multirow[t]{2}{*}{ Week 5} & Ganciclovir & 13 & $5(38)$ & \multirow[t]{2}{*}{0.261} \\
\hline & & Combination & 4 & $0(0)$ & \\
\hline & \multirow[t]{2}{*}{ Week 6} & Ganciclovir & 13 & $3(23)$ & \multirow[t]{2}{*}{1.000} \\
\hline & & Combination & 6 & $2(33)$ & \\
\hline
\end{tabular}

Description: Analysis was done using Fisher's exact test $(n=9)$ at its peak in week 4 . As shown in Table 3, there was no statistically significant difference $(p>0.05)$ in the incidence of side effects of therapy related to decreased kidney function between each regimen during the monitoring period of the therapy.

To further examine the correlation of laboratory examinations to the duration of therapy, Table 4 summarizes the results of laboratory tests before and during therapy monitoring. There were no significant differences during therapy monitoring for hemoglobin $(\mathrm{p}=0.184)$, neutrophil $(p=0.556)$, thrombocyte $(p=0.366)$, creatinine serum $(p=0.929)$, and blood urea nitrogen $(p=0.093)$.

As shown in Table 5, patients with ganciclovir therapy regimen mostly completed the 6-week therapy $(n=21,95 \%)$ while patients with the combination regimen mostly had treatment dropout $(n=16,55 \%)$. This statistical test showed a significant difference $(\mathrm{p}<0.001)$ between each group. No valganciclovir therapy regimen experienced any dropout during therapy since the number of subjects was too small; it was not statistically analyzed.

\section{DISCUSSION}

Cholestasis due to CMV hepatitis can cause intrahepatic and extrahepatic destructive inflammation which results in irreversible fibrosis and 
Table 4: Correlation between results of monitoring laboratory examinations with the duration of therapy

\begin{tabular}{|c|c|c|c|c|c|c|c|c|}
\hline \multirow[t]{2}{*}{ Variable } & \multicolumn{7}{|l|}{ Week } & \multirow[t]{2}{*}{ p-value } \\
\hline & Baseline & 1 & 2 & 3 & 4 & 5 & 6 & \\
\hline \multicolumn{9}{|c|}{ Hemoglobin (g/dL) } \\
\hline $\mathrm{n}$ & 53 & 50 & 45 & 33 & 31 & 25 & 30 & \multirow{3}{*}{$0.184^{\mathrm{a}}$} \\
\hline Mean & 11.0 & 10.4 & 10.4 & 11.1 & 10.8 & 10.3 & 10.4 & \\
\hline SD & 1.9 & 1.5 & 1.4 & 1.7 & 1.7 & 1.8 & 1.6 & \\
\hline \multicolumn{9}{|c|}{ Neutrophil $\left(/ \mathrm{mm}^{3}\right)$} \\
\hline $\mathrm{n}$ & 49 & 44 & 43 & 32 & 31 & 24 & 28 & \multirow{3}{*}{$0.556^{\mathrm{b}}$} \\
\hline Median & 3210 & 3032 & 3038 & 3105 & 3600 & 3480 & 2495 & \\
\hline $\mathrm{SD}$ & 3345 & 2034 & 2207 & 2029 & 2237 & 2536 & 1643 & \\
\hline \multicolumn{9}{|c|}{ Thrombocyte $\left(/ \mathrm{mm}^{3}\right)$} \\
\hline $\mathrm{n}$ & 53 & 50 & 45 & 33 & 31 & 25 & 30 & \multirow{3}{*}{$0.366^{\mathrm{a}}$} \\
\hline Mean & 352,962 & 333,100 & 336,800 & 366,909 & 367,516 & 327,400 & 297,167 & \\
\hline SD & 132,552 & 118,978 & 115,738 & 158,563 & 157,501 & 136,058 & 127,070 & \\
\hline \multicolumn{9}{|c|}{ Creatinine (mg/dL) } \\
\hline $\mathrm{n}$ & 42 & 28 & 36 & 19 & 22 & 17 & 21 & \multirow[b]{2}{*}{$0.929^{\mathrm{b}}$} \\
\hline Median & 0.22 & 0.19 & 0.22 & 0.21 & 0.22 & 0.19 & 0.17 & \\
\hline \multicolumn{9}{|c|}{ Blood urea nitrogen (mg/dL) } \\
\hline $\mathrm{n}$ & 42 & 28 & 36 & 19 & 21 & 17 & 21 & \multirow{3}{*}{$0.093^{\mathrm{b}}$} \\
\hline Median & 14 & 14 & 14 & 13 & 19 & 17 & 17 & \\
\hline SD & 7 & 6 & 6 & 8 & 9 & 7 & 8 & \\
\hline
\end{tabular}

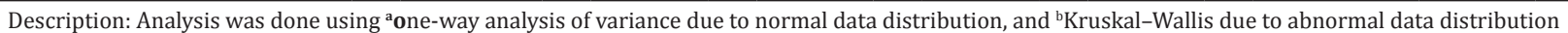

Table 5: Correlation of therapeutic regimen with patient's compliance

\begin{tabular}{llll}
\hline Compliance & $\begin{array}{l}\text { Ganciclovir } \\
(\mathbf{n = 2 2})\end{array}$ & $\begin{array}{l}\text { Combination } \\
\text { (n=29) }\end{array}$ & p-value \\
\hline Finishing 6-week therapy & $21(95 \%)$ & $13(45 \%)$ & $<0.001$ \\
Dropout & $1(5 \%)$ & $16(55 \%)$ & \\
\hline
\end{tabular}

Description: Analysis was done using the Fisher's exact test

cirrhosis $[20,21]$. The number of subjects with cholestasis in this study is different from the study conducted in Israel, where cholestasis was only found in $3 \%$ of the CMV infection pediatric patients who received therapy. This is due to the identification and monitoring of patients with CMV infection has been carried out much better in developed countries compared to developing countries like Indonesia so that disease progression may be prevented [15].

In addition to the involvement of the hepatobiliary system, this study also found many subjects had central nervous system involvement in the form of microcephaly. This is often found in moderate-grade symptomatic CMV infection. Symptomatic CMV infection is often found when viral transmission occurs vertically during pregnancy and horizontally in immunocompromised patients [22]. Symptomatic CMV infection with any involvement of the central nervous system or severe focal organ disease is an indication of ganciclovir therapy $[7,14]$. Ganciclovir provides short-term clinical improvement and provides better neurological development [6,7].

Two patients in the study used valganciclovir therapy regimen because intravenous ganciclovir was not available at the time of treatment so it was decided to use oral valganciclovir for 6 weeks instead. Valganciclovir is a prodrug of ganciclovir hydrolyzed in intestinal and liver epithelial cells to become its active form. This drug is a potent antiviral which has oral bioavailability equivalent to intravenous ganciclovir $[5,9,10]$.

In this study, the combination regimen was the most widely used. The high cost of care, the longer length of stay of inpatient treatment, and the difficulty of using long-term peripheral venous access in patients receiving intravenous ganciclovir for 6 weeks became the consideration to use another efficient yet more convenient alternative. Kadambari et al. recommended the use of a combination therapy regimen using intravenous ganciclovir for 2-3 weeks at the start of therapy and continued with oral valganciclovir if there was difficulty in administering intravenous ganciclovir for 6 weeks, as applied to the majority of subjects in this study [7,11-13]

In this study, there were no differences in the incidence of side effects of neutropenia, thrombocytopenia, anemia, increased creatinine serum, and increased blood urea nitrogen between regimens which were statistically significant. Monitoring from week to week also had shown no significant side effects. Several studies showed that side effects can be found at the beginning of therapy so that rigorous laboratory monitoring needs to be done. Side effects are said to be non-permanent if the dose is lowered or therapy is stopped [23]. Risk factors for myelosuppression-related side effects for ganciclovir include hyperbilirubinemia, high viral load, patients receiving chemotherapy, and low creatinine clearance [24]. The current recommendation is to stop the therapy temporarily if neutrophil $<500 / \mathrm{mm}^{3}$ until neutrophil returns to $>750 / \mathrm{mm}^{3}$ [7]. In this study, the lowest neutrophil value never reached $<500 / \mathrm{mm}^{3}$; therefore, it did not affect the administration of therapy.

The results of this study were in accordance with a study conducted in Turkey, where no side effects of therapy were found related to the therapeutic regimen or duration of the therapy [25]. This may be due to side effects of therapy which are more likely to be high dose related and found in immunocompromised patients or newborns [23]. Since majority of the subjects in this study were infants aged 1-3 months with excluded immunocompromised conditions, it may be the reason for this result. However, studies on these side effects are still a few, with limited population and varying results; therefore, further research is still needed $[11,12,15,16]$

The dropout rate was found significantly higher in the group of combination regimen in this study. These results differ in studies comparing ganciclovir and valganciclovir regimens in CMV recipients of organ transplant recipients where compliance rates were quite good in all groups [12]. Several things thought to be the cause of the high dropout rate include patients living outside of Bandung city making it difficult to do weekly check-ups and low level of socioeconomic or education of patient's family.

This study is the first study that the authors are well aware of examining and comparing any side effects of ganciclovir and/or valganciclovir with the regimen and duration of treatment for pediatrics with CMV infection in developing countries such as Indonesia. However, there are still some limitations to the study, such as the retrospective design of the 
study due to a limited number of subjects, possible confounding factors that can affect laboratory results during therapy, and lack of further monitoring to find out any long-term side effects after the completion of the therapy. Further, research with a prospective research design is needed so that any confounding factors can be reduced to see a further association of the regimen or duration of therapy with therapeutic side effects.

\section{CONCLUSION}

No side effects were found to be related with therapeutic regimen or duration of therapy in pediatric patients with CMV infection given ganciclovir or combination regimen in this study. The choice of regimen can be adjusted to the existing needs while maintaining close monitoring to keep the patient's compliance, especially for those using the combination regimen.

\section{ACKNOWLEDGMENT}

We would like to show our gratitude to Sri Endah Rahayuningsih, Eddy Fadlyana, and Anggraini Alam for comments that greatly improved the manuscript.

\section{AUTHORS' CONTRIBUTIONS}

All authors in this manuscript have contributed to the research. All authors discussed and provided critical feedback on this text.

\section{CONFLICTS OF INTEREST}

The authors declare that there are no conflicts of interest regarding the publication of this paper.

\section{REFERENCES}

1. Knipe D, Howley P, editors. Field's Virology. $5^{\text {th }}$ ed. Philadelphia, PA: Lippincott Williams and Wilkins; 2007.

2. Na SY. Cytomegalovirus infection in infantile hepatitis. Pediatr Gastroenterol Hepatol Nutr 2012;15:91-9.

3. Swanson EC, Schleiss MR. Congenital cytomegalovirus infection: New prospects for prevention and therapy. Pediatr Clin North Am 2013;60:1-17.

4. McMullan BJ, Palasanthiran P, Jones CA, Hall BM, Robertson PW, Howard J, et al. Congenital cytomegalovirus time to diagnosis, management and clinical sequelae in Australia: Opportunities for earlier identification. Med J Aust 2011;194:625-9.

5. Somaji L, Rapolu R. Binding studies of valganciclovir to human serum albumin by multispectroscopic techniques. Int $\mathrm{J}$ Pharm Pharm Sci 2018;10:87-92.

6. Kliegman R, Stanton B, Schor N. Nelson Textbook of Pediatrics. $20^{\text {th }}$ ed. Philadelphia, PA: Elsevier; 2016.

7. Kadambari S, Williams EJ, Luck S, Griffiths PD, Sharland M. Evidence based management guidelines for the detection and treatment of congenital CMV. Early Hum Dev 2011;87:1-6.
8. Patel R, Patel G, Gajra B, Parikh R. Formulation of optimization and characterization of ganciclovir loaded dry chitosan nanoparticles. Asian J Pharm Clin Res 2017;10:295-9.

9. Kimberlin D, Jester P, Sanchez, Ahmed A, Boger R, Michaels N. Valganciclovir for symptomatic congenital cytomegalovirus disease. N Engl J Med 2015;372:933-43.

10. Napoleon AA, Angajala G, Kumar R. Novel synthesis of process related impurities of valganciclovir hydrochloride. Int J Pharm Pharm Sci 2016;8:29-31.

11. Ozkan T, Mistik R, Dikici B, Nazlioglu H. Antiviral therapy in neonatal cholestatic cytomegalovirus hepatitis. BMC Gastroenterol 2007;7:1-5.

12. Asberga A, Humar A, Rollage $H$, Jardined AG, Mouase $H$, Pescovitz MD, et al. Oral valganciclovir is noninferior to intravenous ganciclovir for the treatment of cytomegalovirus disease in solid organ transplant recipients. Am J Transplant 2007:7:2106-13.

13. Asberg A, Humar A, Rollag H, Jardine A, Kumar D, Aukrust P, et al. Lessons learned from a randomized study of oral valganciclovir versus parenteral ganciclovir treatment of cytomegalovirus disease in solid organ transplant recipients: The victor trial. Clin Infect Dis 2016;1:1154-60.

14. Gandhi R, Fernandez-Alvarez JR. Management of congenital cytomegalovirus infection: An evidence-based approach. Acta Pediatr 2010;99:509-15.

15. Bilavsky E, Schwarz M, Bar-Sever Z, Pardo J, Amir J. Hepatic involvement in congenital cytomegalovirus infection-infrequent yet significant. J Viral Hepat 2014;22:1-6.

16. Doan TT, Phung TT, Pham HV, Pham SH, Nguyen LT. Effect of ganciclovir for the treatment of severe cytomegalovirus-associated pneumonia in children without a specific immunocompromised state. BMC Infect Dis 2013;13:424.

17. Rosal TD, Baquero-Artigao F, Blázquez D, Noguera-Julian A, Moreno-Pérez D, Reyes A, et al. Treatment of symptomatic congenital cytomegalovirus infection beyond the neonatal period. J Clin Virol 2012;55:72-4.

18. Prasetyo D. Update Diagnostik Dan Tatalaksana Ikterik Pada Bayi. Pediatric Update 2015. Bandung: Fakultas Kedokteran Universitas Padjadjaran; 2015.

19. Amita D, Balaji O, Patil N. Valganciclovir-induced thrombocytopenia: A case report. Asian J Pharm Clin Res 2017;10:3-4.

20. Young S, Azzam RK. Infantile cholestasis approach and diagnostic algorithm. In: Guandalini, Dhawan A, Branski D, editors. Textbook of Pediatric Gastroenterology, Hepatology and Nutrition. Switzerland: Springer Inc.; 2016. p. 625-31

21. Glaser SS, Gaudio E, Miller T, Alvaro D, Alpini G. Cholangiocyte proliferation and liver fibrosis. Expert Rev Mol Med 2009;25:e7.

22. Plosa EJ, Esbenshade JC, Fuller MP, Weitkamp JH. Cytomegalovirus Infection. Pediatr Rev 2012;33:155-63.

23. Buonsenso DS, Gargiullo L, Ceccarelli M, Ranno O, Valentini P. Congenital cytomegalovirus infection: Current strategies and future perspectives. Eur Rev Med Pharmacol Sci 2012;16:919-35.

24. Matsumoto K, Shigemi A, Ikawa K, Kanazawa N, Fujisaki Y, Morikawa N, et al. Risk factors for ganciclovir-induced thrombocytopenia and leukopenia. Biol Pharm Bull 2015;38:235-8.

25. Tezer H, Secmeer G, Kara A, Ceyhan M, Cengiz AB, Devrim I, et al. Cytomegalovirus hepatitis and ganciclovir treatment in immunocompetent children. Turk J Pediatr 2008;50:228-34. 